\title{
HUBBLE SPACE TELESCOPE OBSERVATIONS OF THE HD 202628 DEBRIS DISK
}

\author{
John E. Krist ${ }^{1}$, Karl R. Stapelfeldt ${ }^{2}$, Geoffrey Bryden ${ }^{1}$, and Peter Plavchan ${ }^{3}$ \\ ${ }^{1}$ Jet Propulsion Laboratory, California Institute of Technology, 4800 Oak Grove Drive, Pasadena, CA 91109, USA \\ ${ }^{2}$ Laboratory for Exoplanets and Stellar Astrophysics, Code 667, NASA Goddard Space Flight Center, Greenbelt, MD 20771, USA \\ ${ }^{3}$ NASA Exoplanet Science Institute, California Institute of Technology, 770 S Wilson Ave., Pasadena, CA 91125, USA \\ Received 2012 March 6; accepted 2012 May 10; published 2012 July 6
}

\begin{abstract}
A ring-shaped debris disk around the G2V star HD $202628(d=24.4 \mathrm{pc})$ was imaged in scattered light at visible wavelengths using the coronagraphic mode of the Space Telescope Imaging Spectrograph on the Hubble Space Telescope. The ring is inclined by $\sim 64^{\circ}$ from face-on, based on the apparent major/minor axis ratio, with the major axis aligned along P.A. $=130^{\circ}$. It has inner and outer radii ( $>50 \%$ maximum surface brightness) of $139 \mathrm{AU}$ and $193 \mathrm{AU}$ in the northwest ansae and $161 \mathrm{AU}$ and $223 \mathrm{AU}$ in the southeast $(\Delta r / r \approx 0.4)$. The maximum visible radial extent is $\sim 254 \mathrm{AU}$. With mean surface brightness of $V \approx 24 \mathrm{mag} \mathrm{arcsec}^{-2}$, this is the faintest debris disk observed to date in reflected light. The center of the ring appears offset from the star by $\sim 28 \mathrm{AU}$ (deprojected). An ellipse fit to the inner edge has an eccentricity of 0.18 and $a=158$ AU. This offset, along with the relatively sharp inner edge of the ring, suggests the influence of a planetary-mass companion. There is a strong similarity with the debris ring around Fomalhaut, though HD 202628 is a more mature star with an estimated age of about 2 Gyr. We also provide surface brightness limits for nine other stars in our study with strong Spitzer excesses around which no debris disks were detected in scattered light (HD 377, HD 7590, HD 38858, HD 45184, HD 73350, HD 135599, HD 145229, HD 187897, and HD 201219).
\end{abstract}

Key words: circumstellar matter - stars: individual (HD 202628, HD 377, HD 7590, HD 38858, HD 45184, HD 73350, HD 135599, HD 145229, HD 187897, HD 201219)

Online-only material: color figures

\section{INTRODUCTION}

Circumstellar debris disks, clouds of dust created by collisions of planetesimals such as comets and asteroids, provide evidence that stars are the central hosts of dynamic systems. Disk structures such as clearings, gaps, and asymmetrical dust distributions may indirectly reveal the presence of planets. The most prominent example is the Fomalhaut debris disk (Kalas et al. 2005) and its apparent planetary-mass companion, Fomalhaut $b$ (Kalas et al. 2008; Chiang et al. 2009). The presence of dust around a star is most easily discerned by a measured infrared excess, though the disk is usually unresolved. While still relatively rare, resolved images of nearby debris disks spanning the millimeter to visible wavelength range have substantially increased in recent years, especially with the use of the $H u b$ ble, Spitzer, and Herschel space telescopes. The dust is often concentrated in a ring, as with Fomalhaut (Kalas et al. 2005), HD 4796 (Schneider et al. 2009), and HD 207129 (Krist et al. 2010). This makes detection easier because the surface brightness is greater than it would be in a more extended disk (e.g., $\beta$ Pictoris).

In an effort to expand the number of resolved debris disks, which so far number around 20, we undertook an imaging survey using the Hubble Space Telescope (HST). For a list of candidate stars with Spitzer-measured infrared excesses and emission ratios of $L_{\text {dust }} / L_{\text {star }}>10^{-4}$, we predicted the detectability of a ring-shaped disk around each assuming a width of $\Delta r / r=0.2$, an albedo of 0.1 , a canonical grain size distribution, and fractional scattered light brightness based on the $L_{\text {dust }} / L_{\text {star }}$. Ten nearby, solar-type targets with predicted inner radii outside of the inner working angle of the HST Space Telescope Imaging Spectrograph (STIS) coronagraph were chosen. Our goal was to combine the dust distribution seen in the HST images with Spitzer measurements to derive the actual grain properties (spatial distribution, albedo, scattering phase function, grain size distribution, etc.).

Of the 10 stars in our program, we have imaged a disk around only 1, HD 202628 (HIP 105184, GJ 825.2, SAO 230622), a G2V star $(V=6.75)$ located at 24.4 pc. Koerner et al. (2010) measured a $\lambda=70 \mu \mathrm{m}$ excess $(17 \times$ the photosphere) using Spitzer, deriving $L_{\text {dust }} / L_{\text {star }}=1.4 \times 10^{-4}$. A central clearing is indicated by the lack of any $24 \mu \mathrm{m}$ excess that would be emitted by warmer dust near the star. Using the assumptions discussed above, we predicted an inner disk radius of $80 \mathrm{AU}$ (3".4).

\section{OBSERVATIONS AND DATA PROCESSING}

STIS observations of HD 202628 were taken on 2011 May 15 as part of $H S T$ program 12291 (file prefixes obhs 05 and obhs06). As with all of the targets in this program, it was imaged over two consecutive orbits with the telescope rolled by $28^{\circ}$ between them. Each orbit began with a coronagraphic target acquisition image, and then the star was placed behind the occulting wedge (WEDGEA1.8 aperture position, 0.'9 mask radius). Eight exposures of $282 \mathrm{~s}$ each (2256 s total per orbit) were taken. The relatively short exposure times were required to reduce saturation. We initially used combined and cosmic-rayrejected images (_crj.fits files) from the $H S T$ calibration pipeline but found that the drift in the star position between exposures was large enough to create significant artifacts after point-spread function (PSF) subtraction, since the drift is not accounted for by the pipeline. We therefore manually registered each exposure to the first image in the first orbit to within 0.05 pixels ( 3 mas) using damped sinc interpolation. This was done by adjusting the shift of each image until the radial streaks from the stellar PSF were visually minimized. We combined the results for each orbit using our own cosmic-ray rejection routine. 


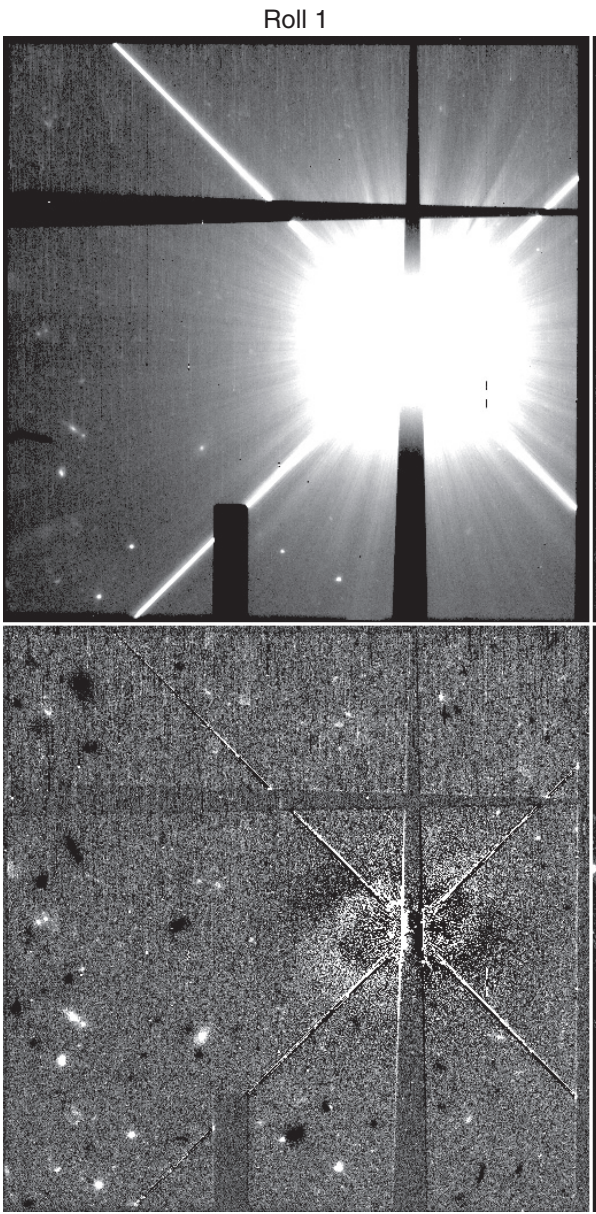

Roll 1 - Roll 2

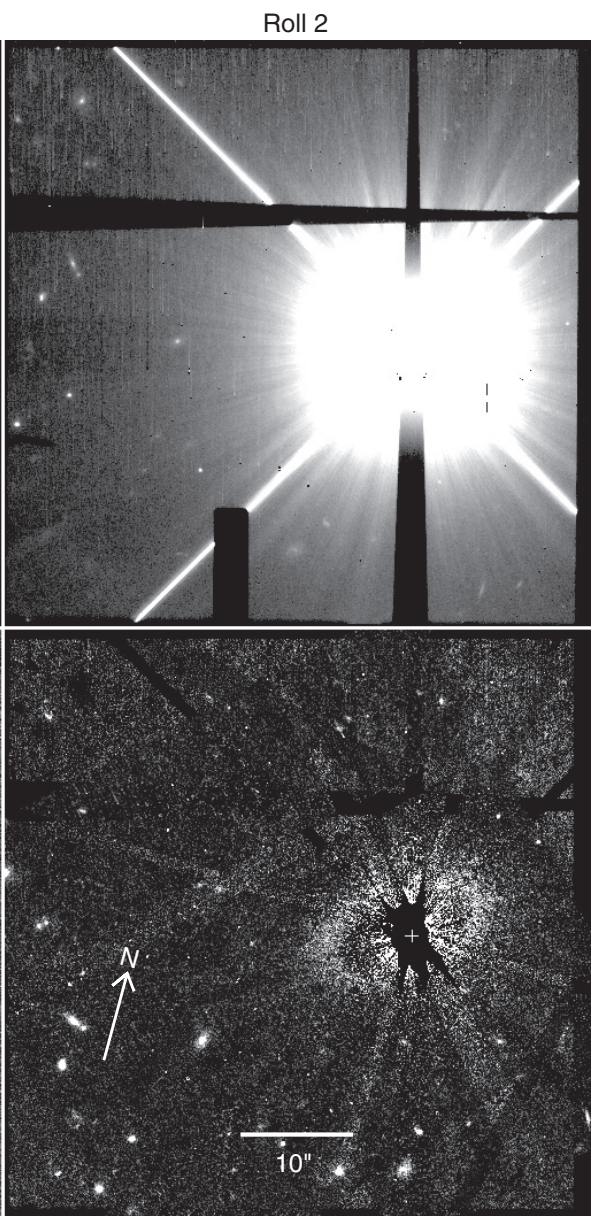

Iterative Algorithm

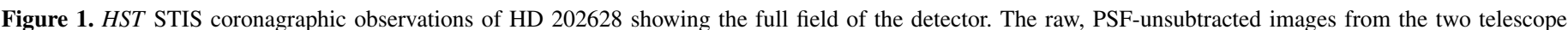

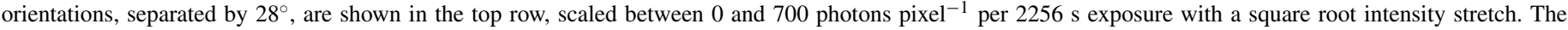

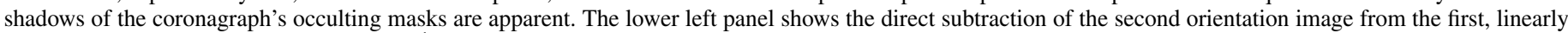

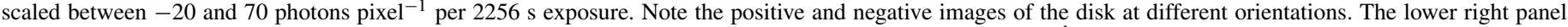

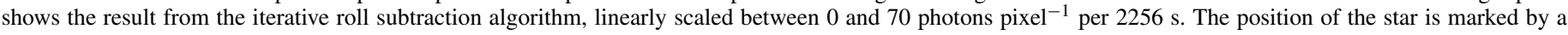
cross.

Despite using the coronagraphic mode, scattered and unsuppressed diffracted light from the star dominates the images, so PSF subtraction is required. Taking advantage of the relative stability of $H S T$, this is usually done by observing another star of similar color and subtracting its scaled and registered image. STIS allows for only unfiltered imaging in its coronagraphic mode, so the effective wavelength bandpass is set by the CCD detector and is therefore quite broad (250-1100 nm). Because the fine PSF structure is dependent on object color, even minute color differences between the target and reference PSFs can introduce substantial residual artifacts that might overwhelm a faint disk. We therefore chose to not observe a reference star and instead used the iterative roll subtraction algorithm (Figure 1) that was successfully applied to HST observations of the HD 207129 debris disk (Krist et al. 2010). This method dispenses with the need for another orbit to obtain a reference PSF and avoids any color mismatch issues. The main drawback is that any disk seen in a face-on (or nearly so) orientation will essentially subtract itself out. Krist et al. demonstrated via modeling that this method produces reliable results for inclined disks. To set the roll pivot point, the position of the star behind the mask was derived from the intercept of the diffraction spikes; experiments indicate that the position is accurate to with 0.1 pixel ( 5 mas).
As a comparison, we performed PSF subtraction using images of other stars from our program, HD 45184 and HD 73350 (stellar properties are listed in the Appendix). These images were registered and scaled in intensity to match HD 202628 prior to subtraction. The results using these reference PSFs (Figure 2) show larger residuals, especially near the star, and worse definition.

We note that there are dozens of small galaxies distributed over the field, some barely resolved. They introduce a significant source of confusion for planet searches in this system. A common proper motion determination of any companion candidate will be a necessity. Fortunately, this star has a high proper motion (241 mas $\left.\mathrm{yr}^{-1}\right)$, so it would not take long to show that a source is comoving. There are no obvious point sources inside the disk or within $4^{\prime \prime}$ of the outer edge.

\section{RESULTS}

\subsection{General Disk Morphology}

As shown in Figure 1, positive and negative images of a disk, along with background sources, are apparent when the image from the second roll is directly subtracted from the one from the 

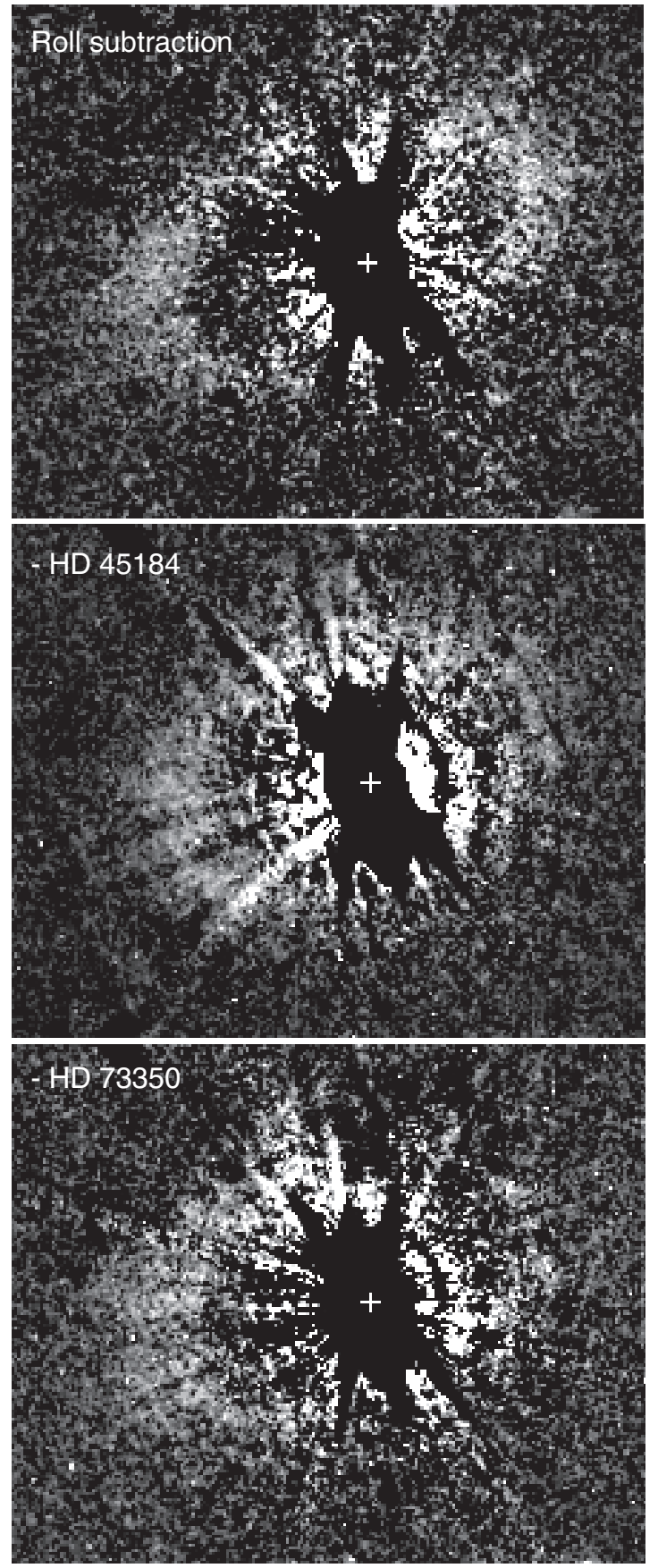

Figure 2. STIS coronagraphic observation of HD 202628 with the stellar PSF subtracted using (top) the iterative roll subtraction algorithm (middle), an image of HD 45184, and (bottom) an image of HD 73350. Same orientation as Figure 1.

first. Application of the iterative subtraction algorithm reveals that the disk is a ring inclined from face-on by $\sim 64^{\circ}$ (based on the apparent major/minor axes ratio) with the apparent major axis aligned along P.A. $=130^{\circ}$. Most of the disk along the minor axis is obscured by large subtraction residuals, the STIS occulter, and the HST diffraction spikes. Within $r<3$ ".5 (85 AU) there is a halo of unsubtracted starlight; such residuals are expected due to PSF mismatches caused by time-dependent telescope aberrations and pointing errors (Krist 2004).

The ring is asymmetric. The inner and outer apparent edges of the northwest ansa are at 5..8 (142 AU) and 8.7 (212 AU), respectively. In the SE ansa they are at 6.'6 (162 AU) and 10'.4

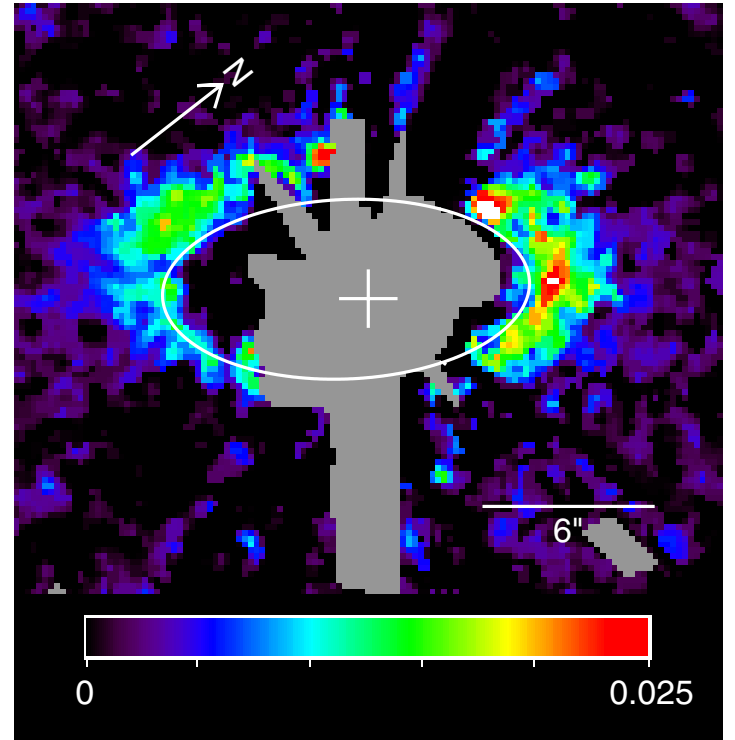

Figure 3. Surface brightness map of the HD 202628 disk. The image has been median filtered and rebinned to lower sampling to reduce noise. The position of the star is marked by a cross. An ellipse, fitted to the inner ring edge in the original image, is superposed. Known regions of high residuals or obscurations are colored gray. The measured count rate (photons per second per $0^{\prime \prime} 051 \times 0.0^{\prime \prime} 0.51$ pixel) is shown in the linear intensity scale legend $\left(0.025\right.$ photons s ${ }^{-1}$ pixel $^{-1}$ is equivalent to $V=23.6$ mag $\operatorname{arcsec}^{-2}$, assuming neutral scattering).

(A color version of this figure is available in the online journal.)

(254 AU). The outer radius is noise limited, so based on the apparent image the fractional width is $\Delta r / r \approx 0.4$.

There are azimuthal brightness asymmetries, as shown in Figure 3. The ring appears clumpy due to the noise, but it is probably azimuthally smooth. The per pixel signal-to-noise ratio is about 1.0 in the ansae; the mean surface brightness in the SE ansa is $\sim 80 \times$ less than the PSF at the same location before subtraction. We would not expect any significant amount of material within the ring interior due to the lack of $24 \mu \mathrm{m}$ emission (Koerner et al. 2010). Without any color information, we assumed neutral scattering by the dust and measured peak and mean surface brightnesses, respectively, of $V=23.6$ and $24.0 \mathrm{mag} \operatorname{arcsec}^{-2}( \pm 0.2 \mathrm{mag})$ in the NW ansa. ${ }^{4}$ The SE ansa is about half as bright. Visible portions of the NE section of the ring are about $20 \%-50 \%$ brighter than the opposite side. For comparison, the Fomalhaut ring has a surface brightness of 21.5 mag $\operatorname{arcsec}^{-2}$ (Kalas et al. 2005) and HD 207129's is 23.7 (Krist et al. 2010).

\subsection{Ring Eccentricity}

The ring is eccentric, and the offset of the ring center from the star is apparent in Figure 4. Due to the low signal that prevented a reliable least-squares fit, we visually fitted an inclined ellipse to the ring inner edge while fixing one focus to the position of the star. The size of the ring, its aspect ratio, position angle (P.A.), and two-dimensional offset of the star from the center of the ellipse provide five independent observables. These are sufficient to constrain the five parameters needed for the model ellipse: semimajor axis, eccentricity, inclination, major axis P.A., and argument of periapse relative to the sky plane. Trial

\footnotetext{
4 Without a direct measurement of the star's brightness for photometric calibration, we used the flux rate provided by the STIS Exposure Time Calculator of $5.30 \times 10^{7}$ photons s $^{-1}$ for a G2V $V=6.75$ star.
} 

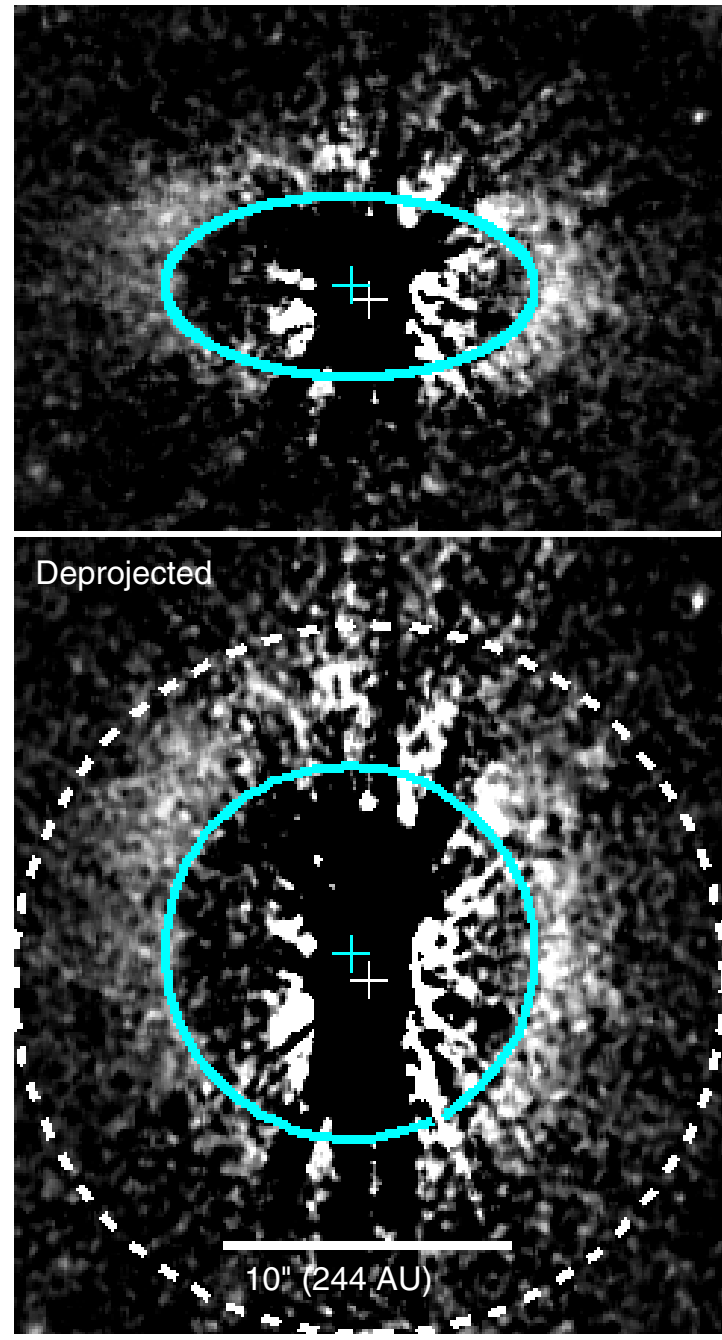

Figure 4. Direct (top) and deprojected (bottom) images of the HD 202628 disk, shown with linear intensity stretches. P.A. $=40^{\circ}$ is toward the top. The position of the star is marked with a white (lower right) cross. An ellipse fitted to the ring inner edge (same ellipse as is shown in Figure 3 ) is plotted as a solid blue line, and the ellipse geometric center is marked with a blue (upper left) cross. A $300 \mathrm{AU}$ radius dashed circle centered on the star is shown for comparison. Interior to the ring there are large PSF subtraction residuals, and the occulting mask obscures portions of the ring. Both images have been median filtered and $2 \times 2$ rebinned to lower sampling to improve the signal.

(A color version of this figure is available in the online journal.)

model runs showed that the semimajor axis, P.A., and inclination are strongly constrained, while the eccentricity and argument of periapse are slightly degenerate with each other. The latter interaction arises in this case because the stellar offset from the ring center is aligned along its projected minor axis, rather than along the major axis as in the case of Fomalhaut (Kalas et al. 2005).

The best-fit parameters are an inner edge semimajor axis of $158 \mathrm{AU}$ at P.A. $=134^{\circ}$, inclination of $61^{\circ}, e=0.18$, and ellipse major axis aligned $60^{\circ}$ behind the plane of the sky. While a smaller eccentricity combined with a major axis close to the sky plane would adequately fit the observed image, the parameters reported provided a much better fit to the deprojected image of the system. We estimate a \pm 0.02 uncertainty in $e$. The geometric center of the ellipse is offset from the star in the non-deprojected image by $19.5 \mathrm{AU}$ (16.0 AU along P.A. $=130^{\circ}$ and $11.3 \mathrm{AU}$ along P.A. $=40^{\circ}$ ). Deprojected, the offset is $28.4 \mathrm{AU}$ (16.0 AU along P.A. $=130^{\circ}$ and $23.4 \mathrm{AU}$ along P.A. $=40^{\circ}$ ).

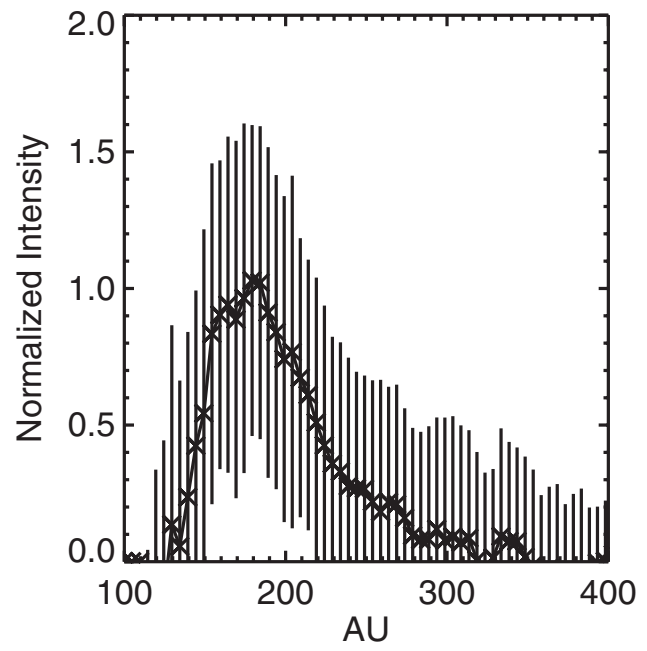

Figure 5. Radial surface brightness profile of the HD 202628 ring measured using the azimuthal median within $90^{\circ}$ sectors centered on P.A. $=130^{\circ}$ and $210^{\circ}$ in the filtered, deprojected image. The root-mean-square error bars are included.

\subsection{Spitzer Observations}

Koerner et al. (2010) observed HD 202628 with Spitzer/ MIPS, finding a strong $70 \mu \mathrm{m}$ excess but only a photospheric flux density at $24 \mu \mathrm{m}$. An upper limit to the $24 \mu \mathrm{m}$ excess can be assumed to be $3 \times$ the uncertainty in the absolute calibration, which is approximately $10 \%$ of the $24 \mu \mathrm{m}$ flux density or $3.9 \mathrm{mJy}$. This, in combination with the $70 \mu \mathrm{m}$ excess of $100 \mathrm{mJy}$, implies a dust temperature upper limit of $\sim 65 \mathrm{~K}$. If a minimum grain size of a few microns and silicate emissivities are assumed (following Krist et al. 2010), the dust would need to be located at least $80 \mathrm{AU}$ from the star to reproduce the Spitzer photometry. The observed ring inner radius of $160 \mathrm{AU}$ implies a substantially colder dust temperature than the current Spitzer limit. Upcoming Herschel observations should clarify the spectral energy distribution and characteristic dust temperature for the disk.

The ring's diameter of $\sim 14^{\prime \prime}$ (300 AU) observed with STIS is large enough to produce a resolved $70 \mu \mathrm{m}$ source to Spitzer/MIPS. Koerner et al. (2010) do not report any size information for their $70 \mu \mathrm{m}$ detections, so we investigated the source size in post-BCD mosaics retrieved from the Spitzer Heritage Archive. A two-dimensional elliptical Gaussian fit to the $70 \mu \mathrm{m}$ source has a full width at half-maximum of $22^{\prime \prime} .1 \times$ 19'. 1 extended along P.A. $138^{\circ}$. Quadrature subtraction of the nominal MIPS $70 \mu \mathrm{m} \mathrm{16"} \mathrm{beam} \mathrm{suggests} \mathrm{an} \mathrm{intrinsic} \mathrm{source} \mathrm{size}$ of $15^{\prime \prime} \times 10^{\prime \prime}$, consistent with the inclined ring seen in scattered light and elongated at essentially the same P.A. A small 0 .'6 offset between the centers of the 24 and $70 \mu \mathrm{m}$ sources is not significant given the $70 \mu \mathrm{m}$ signal-to-noise ratio of 11 (Koerner et al. 2010), so an assessment of a possible pericenter glow (Wyatt et al. 1999) awaits more sensitive Herschel data.

\section{DISCUSSION \\ 4.1. Radial Profile}

Figure 5 shows the ring's radial (relative to the disk center) surface brightness profile. It was derived from the filtered, deprojected image by computing the median value at each radius within the two $90^{\circ}$ sectors on opposite sides of the star aligned along the apparent major axis of P.A. $=130^{\circ}$ (horizontal 

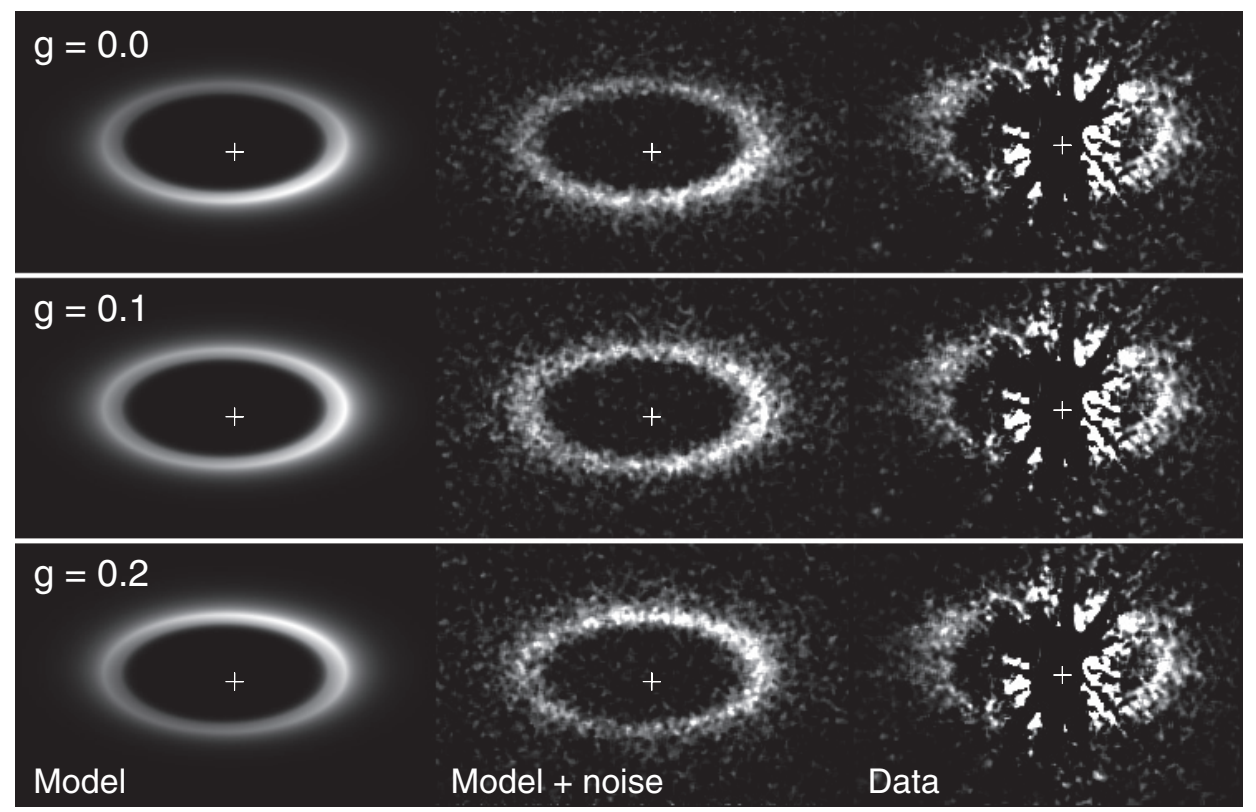

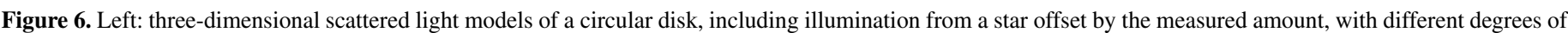

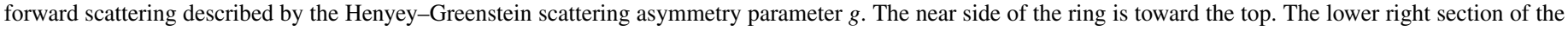

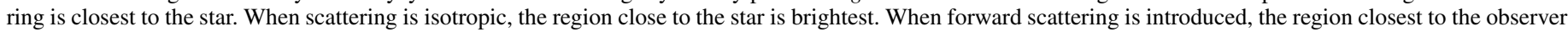

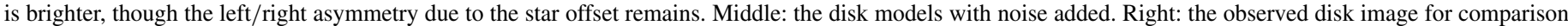

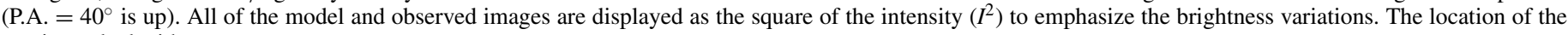
star is marked with a cross.

axis in the deprojection). Note that no correction for azimuthal brightness variations was applied. The azimuthal root-meansquare (rms) values were concurrently determined. The radius was measured from the fitted ellipse's geometric center. The low signal-to-noise ratio of the image results in large error bars, but a rapid dropoff along the inner edge is evident and indicates a rather sharp truncation. However, we cannot discern whether the slope is due to the difference between a mid-plane radial density variation, the vertical disk structure seen integrated along the lines of sight, or a combination of both, though for a geometrically and optically thin disk the projection effect would be insignificant. The profile extends above the field noise (which has an rms equivalent surface brightness of $\sim 24.7 \mathrm{mag} \mathrm{arcsec}^{-2}$ ) over 120-300 AU ( $\Delta r / r \approx 0.7)$. Between 150 and 220 AU $(\Delta r / r=0.4)$ the intensity is $>50 \%$ maximum brightness, which is commonly used to define widths of ring-shaped disks. Rough estimates of radial power laws that describe the different zones of the surface brightness profile were determined: $r^{12}$ (100 AU $<r<156 \mathrm{AU}), r^{2}$ (156 AU $\left.<r<182 \mathrm{AU}\right), r^{-4.7}$ $(r>182 \mathrm{AU})$. The true outer radius is indeterminate due to the noise.

\subsection{Azimuthal Brightness Variations}

The western inner edge of the ring is closest to the star. A line drawn through the star and along P.A. $=130^{\circ}$, the apparent major axis of the ring (i.e., a horizontal line in Figure 4), intercepts the ring (as located by the fitted ellipse) at distances 137 (NW) and 172 (SE) AU from the star. In an azimuthally uniform disk, the brightness at the SE intercept of the line and ring should be $63 \%$ of that in the NW due to the difference in distances from the star. The SE is actually more than $50 \%$ fainter, so the ring probably does not have an azimuthally uniform dust distribution. Since both locations are at the same scattering angle, the degree of forward scattering does not affect the ratio. However, if we assume isotropic scattering, then the SW side, being closer to the star, should be brighter than the NE, but it is not. If we assume that the NE side of the ring is closest to us, forward scattering could cause the NE/SW brightness asymmetry.

To demonstrate this qualitatively and to constrain the degree of forward scattering, we generated three-dimensional-scattered light models. For simplicity, a circularly symmetric, azimuthally uniform disk with the measured offset from the star was implemented (the deviation of an elliptical ring from a circular one would not qualitatively change the results; the bulk of the illumination effect is due to the ring offset from the star). The model's dust distribution was specified by the three power laws described previously (though with +2 added to the exponents to account for the additional $r^{-2}$ stellar illumination falloff in the surface brightness). Without any strong constraint from the noisy data, an outer disk radius of $450 \mathrm{AU}$ was assumed. We used a flat (non-flaring) disk with a 2 AU scale height (this is largely unimportant for these purposes). The HenyeyGreenstein scattering phase function was used with the amount of forward scattering defined by the parameter $g(0=$ isotropic, $1=$ full forward scattering). To aid visual comparisons with the data, the models were PSF convolved, and then Poisson and read noise was added. Given the poor signal-to-noise level, we did not attempt to fit these models to the data but used them only to provide qualitative comparisons.

The models are shown in Figure 6. The top side of the model ring is closest to us. With isotropic scattering $(g=0)$, regions of the ring closest to the star are brighter. Once forward scattering is introduced, the near side becomes brighter, though the left/right asymmetry remains. It appears from a qualitative comparison with the data that the grains are only mildly forward scattering $(0.15<g<0.25)$.

\subsection{The Age of HD 202628}

Valenti \& Fischer (2005) placed HD 202628 on a theoretical isochrone using its distance and absolute luminosity (assumed 
to be solar) to derive a best-fit age of 5.0 Gyr and an allowable range of 1.8-6.8 Gyr. Holmberg et al. (2009) estimated 5.9 Gyr with an even wider range of 1.6-10.2 Gyr, also based on isochrone fitting. However, the $\mathrm{Ca}$ II $\mathrm{H}$ and $\mathrm{K}$ lines suggest activity consistent with a younger star $\left(\log R_{\mathrm{HK}}^{\prime}=-4.73\right.$; Henry et al. 1996), as does the X-ray emission $\left(\log L_{X}=28.41\right.$; Hünsch et al. 1999). Using the methods described by Plavchan et al. (2009, hereafter P09), we derive ages of 2.3 Gyr using $R_{\mathrm{HK}}^{\prime}$ and $1.2 \mathrm{Gyr}$ using $L_{X}$. The star is clearly younger than the Sun but older than $1 \mathrm{Gyr}$, so we assume an age of $2.3 \pm 1 \mathrm{Gyr}$. This makes HD 202628 one of the oldest stars with an imaged debris disk.

\subsection{Is the Ring Evidence of a Planet?}

The eccentricity and sharp inner edge of Fomalhaut's ring suggested perturbations from a planetary-mass object (Kalas et al. 2005). Quillen (2006) predicted that a planet should be orbiting just inside the ring edge and with the same eccentricity. The discovery of Fomalhaut $b$ near this predicted location appeared to validate this model (Kalas et al. 2008). The similar ring structures between HD 202628 and Fomalhaut likewise suggest the action of planetary perturbations in this new system. Two noteworthy differences are the higher eccentricity and broader width (in $\Delta r / r$ terms) of HD 202628's ring. The former likely reflects a higher eccentricity for the perturbing planet. A possible explanation for the latter is suggested by the Chiang et al. (2009) models for the spatial distribution of small dust particles derived from parent bodies in an eccentric ring. Their Figure 3 shows how the steepness of the ring inner edge can be produced by either a lower-mass planet close to the ring or a higher-mass planet at a greater interior distance. Predictions for the outer profile differ substantially between these two scenarios, however, depending on mass and orbital radius. Specific simulations for HD 202628 are needed, but a semimajor axis of $\sim 120 \mathrm{AU}$ and mass $<10 M_{\text {Jupiter }}$ seem plausible.

There are significant limits to detecting planets at large orbital radii $(>100 \mathrm{AU})$ and older than a couple hundred million years in the infrared (due to low thermal emission from mature planets) and in the visible with future space coronagraphs (due to low reflected light contrast, even from giant planets). Debris disk structures are thus critical, indirect indicators of planetary system properties at large radii. The structure of debris rings such as HD 202628 and Fomalhaut indicates that planets must exist at larger scales than previously thought, a significant challenge for planet formation theory. It is believed that in situ core accretion cannot take place rapidly enough at these distances to form a planet before the dissipation of the primordial disk (Dodson-Robinson et al. 2009). In Fomalhaut, HR 8799 (Marois et al. 2008; Su et al. 2009), and now HD 202628, the distant planets are seen or inferred within their circumstellar debris belts-thus indicating that they must have formed from within a circumstellar disk and not (as binary stars do) from adjacent collapsing cloud cores. Both Fomalhaut and HR 8799 are A stars, and their protoplanetary disks would likely have been fairly massive. HD 202628, a G star, would have had a less massive accretion disk, so the presence of a planet even further from the star than those around the A stars would be a mystery. The eccentricity of the purported planet, as suggested by that of the ring, would indicate that it formed closer to the star and then scattered outward by a more massive planet. Such a planet could not have been captured from outside the system, otherwise the bodies creating the debris disk would have been scattered.

\subsection{Grain Properties and Collision Timescales}

The deprojected image was used to estimate a disk/star scattered light fraction. The total flux was measured within the two $90^{\circ}$ sectors centered on P.A. $=130^{\circ}$ and $310^{\circ}$ (along the ring apparent major axis) and the sum doubled to account for the obscured sections of the ring along the line of sight. By this technique, $F_{\text {scat }}=F_{\text {disk }} / F_{\text {star }}=6.8 \times 10^{-6}$ with an estimated error of $\pm 8 \%$. We can make a rough estimate of the albedo by computing the ratio of the scattered light fraction to the sum of scattered and emitted flux fractions $\left(F_{\text {emit }}=L_{\text {dust }} / L_{\text {star }}=1.4 \times 10^{-4}\right.$ from Koerner et al. 2010): $a \approx F_{\text {scat }} /\left(F_{\text {scat }}+F_{\text {emit }}\right) \approx 4.6 \%$. This is quite similar to HD $207129\left(F_{\text {emit }}=L_{\text {dust }} / L_{\text {star }}=7.6 \times 10^{-6}, a \approx 5 \%\right.$; Krist et al. 2010). Note that Fomalhaut has an intrinsically fainter disk $\left(F_{\text {scat }}=9.6 \times 10^{-7}\right)$, but the star is much brighter $(V=1.2)$ so its disk has a higher apparent surface brightness $\left(V \approx 21.5\right.$ mag $\left.\operatorname{arcsec}^{-2}\right)$.

Grain-grain collisions dominate the evolution of the dust in the HD 202628 disk until the grains are ground down to a radiative blowout radius of $\sim 1 \mu \mathrm{m}$ and are expelled from the system. HD 202628 is younger than the Sun with an enhanced X-ray luminosity of $L_{X} / L_{X_{\odot}}=10^{28.41} / 10^{27.35}=11.5$. Using Equation (3) from P09, we estimate a stellar wind with $\sim 26 \times$ the solar wind mass-loss rate, assuming that the radius of HD 202628 is approximately equal to that of the Sun. Using Equations (A10) and (A16) in P09, we estimate the factor $P_{\mathrm{CPR}} \approx 9.7$ that the enhanced stellar wind contributes to shortening the Poynting-Robertson drag timescale. Finally, using Equation (A23) in P09, with $L_{\text {dust }} / L_{\text {star }}=1.4 \times 10^{-4}$, $D_{\text {ring }}=158 \mathrm{AU}, Q_{\text {coll }}=1$, and assuming the mass and luminosity of HD 202628 match the Sun, we estimate that the grain-grain collision timescale is $\sim 100$ times shorter than that for the grains to spiral inward toward the star under the combined effects of stellar wind and Poynting-Robertson drag. Equation (A23) in P09 is independent of factors such as the grain size and density; it assumes a relative disk annulus of 0.1 times the disk diameter, whereas the disk for HD 202628 is four times as thick in annular diameter. Therefore, this timescale ratio is only a lower bound, but we can definitively conclude that grain-grain collisions dominate the dust grain evolution of this disk and that the dust grains originate from the collisions of larger parent bodies within the same annulus (e.g., Kuiper Belt object analogs).

\subsection{Comparisons to Other Ring-like Disks}

There are a variety of ring-shaped debris disks that have been imaged in scattered light. The widths of the rings, the sharpness of their inner and outer edges, their ellipticities (or lack thereof) vary widely. Here, we summarize them to provide some context for the HD 202628 ring. Note that we omit extended disks with central clearings (e.g., $\beta$ Pic).

Of all the other disks, HD 202628's seems most like Fomalhaut's. Fomalhaut's ring has a sharp inner edge with similar surface brightness profiles of $r^{10.9}$ along the inner edge and an outer falloff of $r^{-4.6}$ (Kalas et al. 2005). The Fomalhaut ring is smaller than HD 202628's (113-158 AU; $\Delta r=25$ AU; $\Delta r / r=0.2$; unless otherwise noted, ring sizes given here represent the $>50 \%$ of maximum surface brightness zones), and it has a lower eccentricity $(e=0.11$ versus 0.18$)$ with a smaller star/ring offset (15.3 AU versus $28.4 \mathrm{AU}$ ). The Fomalhaut $L_{\text {dust }} / L_{\text {star }}=8 \times 10^{-5}$ is about half that of HD 202628, and the star is younger $(\sim 200 \mathrm{Myr})$. 
Table 1

Disks with Ring-like Architecture

\begin{tabular}{|c|c|c|c|c|c|c|}
\hline \multirow{2}{*}{$\begin{array}{l}\text { Star } \\
\text { Name }\end{array}$} & \multirow{2}{*}{$\begin{array}{c}\text { Spectral } \\
\text { Type }\end{array}$} & \multirow{2}{*}{$\begin{array}{l}L_{\text {dust }} / L_{\star} \\
\quad \times 10^{-4}\end{array}$} & \multicolumn{3}{|c|}{ Ring Width $^{\mathrm{a}}$} & \multirow[t]{2}{*}{ Comments } \\
\hline & & & $\Delta r(\mathrm{AU})$ & $\Delta r / r$ & $e$ & \\
\hline HD 202628 & G2V & 1.4 & $150-220$ & 0.4 & 0.18 & \\
\hline HD 207129 & G0V & 1.4 & $148-178$ & 0.2 & None & Visible range, not half-brightness \\
\hline Fomalhaut & A3V & 0.8 & $113-158$ & 0.2 & 0.11 & \\
\hline HD 107146 & G2V & 8 & $87-168$ & 0.6 & None & \\
\hline HD 181327 & F5V & 20 & $80-100$ & 0.2 & None $^{b}$ & Halo seen out to $455 \mathrm{AU}$ \\
\hline HR 4796 & $\mathrm{~A} 0 \mathrm{~V}$ & 50 & $65-91$ & 0.3 & 0.07 & \\
\hline HD 92945 & $\mathrm{~K} 1 \mathrm{~V}$ & 8 & $40-63,117^{\mathrm{c}}$ & $0.4,1.0^{\mathrm{c}}$ & None & Two blended rings? \\
\hline
\end{tabular}

Notes. For a complete scattered light debris disk list, see Golimowski et al. (2011).

${ }^{a}$ Unless otherwise noted, ring widths correspond to the region with surface brightness $>50 \%$ of maximum.

b No eccentricity was reported for HD 181327 based on HST/NICMOS observations. However, higher resolution HST /STIS observations have been obtained in GO Program 12228 (PI: Schneider) that are likely to provide a better constraint on eccentricity, though results from that study were not available at the time of writing.

${ }^{c}$ For HD 92945, the first outer radius given is that of the inner ring, the second is of the outer ring; the width ratios are, respectively, for the inner ring only and the combined rings.

HD 181327 (F5V, Schneider et al. 2006; Lebreton et al. 2012) has a ring-shaped disk extending from 80 to $100 \mathrm{AU}$ $(\Delta r=20 \mathrm{AU} ; \Delta r / r=0.2)$, though a faint halo of dust can be seen out to $\sim 455$ AU. This is a much brighter and more massive disk $\left(L_{\text {dust }} / L_{\text {star }}=2 \times 10^{-3}\right)$ than HD 202628's or Fomalhaut's. Curiously, its surface brightness profile is very similar to Fomalhaut's and HD 202628's, with the inner edge rising as $r^{10}$ and the outer disk falling as $r^{-4.7}$ (Lebreton et al. 2012). There is no reported eccentricity.

HR 4796 (A0V) is quite young (8 Myr), and it has one of the brightest debris disks $\left(L_{\text {dust }} / L_{\text {star }}=5 \times 10^{-3}\right)$. Its ringshaped disk is small and narrow $\left(r_{\text {in }} \approx 65 \mathrm{AU}, r_{\text {out }} \approx 91 \mathrm{AU}\right.$; $\Delta r=25 \mathrm{AU} ; \Delta r / r=0.3)$. Both its inner and outer edges are sharp; the cause of the outer edge truncation has yet to be satisfactorily explained, but it has been demonstrated that it cannot be due to a known, nearby stellar companion (Thébault et al. 2010). Recent adaptive optics imaging (Thalmann et al. 2011) verified a 1.2 AU offset along the apparent major axis seen by Schneider et al. (2009), but a $~ 5$ AU offset along the line of sight was also seen. This suggests an eccentricity of $e \approx 0.07$.

HD 207129 (G0V; Krist et al. 2010) has a faint $\left(V=23.7 \mathrm{mag} \operatorname{arcsec}^{-2}\right)$, ring-shaped disk extending over 148-178 AU $(\Delta r=30 \mathrm{AU} ; \Delta r / r \approx 0.2$; note that these are the visible, not $50 \%$ brightness, extents). There is no indication of an offset of the ring or any significant azimuthal density variations. Due to low signal, reliable surface brightness profiles could not be obtained. It has little or no forward scattering.

HD 107146 (G2V; Ardila et al. 2004) has a low-inclination disk with an inner clearing at $r \approx 60 \mathrm{AU}$. The surface brightness increases gradually out to $130 \mathrm{AU}$ and then falls just as gradually further out. The $50 \%$ surface brightness radii are $87 \mathrm{AU}$ and $168 \mathrm{AU}(\Delta r=81 \mathrm{AU} ; \Delta r / r=0.6)$. There is no observed eccentricity.

HD 92945 (K1V; Golimowski et al. 2011) has a disk that extends from $r=43-140$ AU. The inner clearing is not fully defined in the HST images, but there is a density enhancement along the inner edge at 40-70 AU. The outer edge also has enhanced density up to $100 \mathrm{AU}$, beyond which the surface brightness falls off dramatically $\left(\sim r^{-10}\right)$. This may indicate that there are blended inner and outer rings. The disk has no notable eccentricity.

Table 1 summarizes some of the important properties of these disks. There seems little in common among them, except that they are, by selection, rings. The inner radii (based on $>50 \%$ intensity) vary considerably, from 40 to $150 \mathrm{AU}$. The sharp inner edges of some (HD 202628, Fomalhaut, HR 4796, and perhaps HD 181327) are likely defined by the orbital radii and masses of planets that are truncating them. The existence of planets is further bolstered by the eccentricities seen in at least three of the rings (HD 202628, Fomalhaut, and HR 4796). In contrast, HD 107146 has a gentle radial brightness profile without a sharp inner edge. Ertel et al. (2011) and Hughes et al. (2011) suggest that this favors a morphology induced by a collisional cascade of planetesimals unaltered by planetary perturbation. The sharpness of the inner edge of HD 92945 is uncertain due to subtraction residuals near the star (Golimowski et al. 2011) and of HD 207129 due to poor signal (Krist et al. 2010). In two cases, HR 4796 and HD 92945, the outer disk extents are sharply truncated, perhaps due to unseen companions (note that the outer radii of both are smaller than the inner radius of HD 202628). All of the other disks have gradual brightness falloffs with increasing radius, characteristic of grains being blown out by radiation pressure and winds, and, likely in some cases, bumped into higher eccentricities by planets (HD 202628 is uncertain due to poor definition).

\section{CONCLUSIONS}

HD 202628 is one of only three stars, including Fomalhaut and HR 4796, with eccentric, ring-shaped debris disks with sharp inner edges, all signs of planetary tidal interactions. It has the largest and most eccentric ring of the three. It is also the only solar-type star and is much older ( $\sim 2 \mathrm{Gyr})$ than the other two. Based on the previous modeling of the Fomalhaut ring (Quillen 2006; Chiang et al. 2009), the perturbing planet in the HD 202628 system is probably close to, and inside of, the $\sim 158$ AU inner edge. This shows that planets at very large orbital radii are present around not only more massive stars like Fomalhaut, which had presumably more massive accretion disks. This may be evidence that planets may form closer to the stars and get scattered to large distances by other, more massive planets.

We hope to obtain deeper exposures of HD 202628 with HST that would show this faint disk with better definition. These would allow more accurate characterizations of the inner edge 

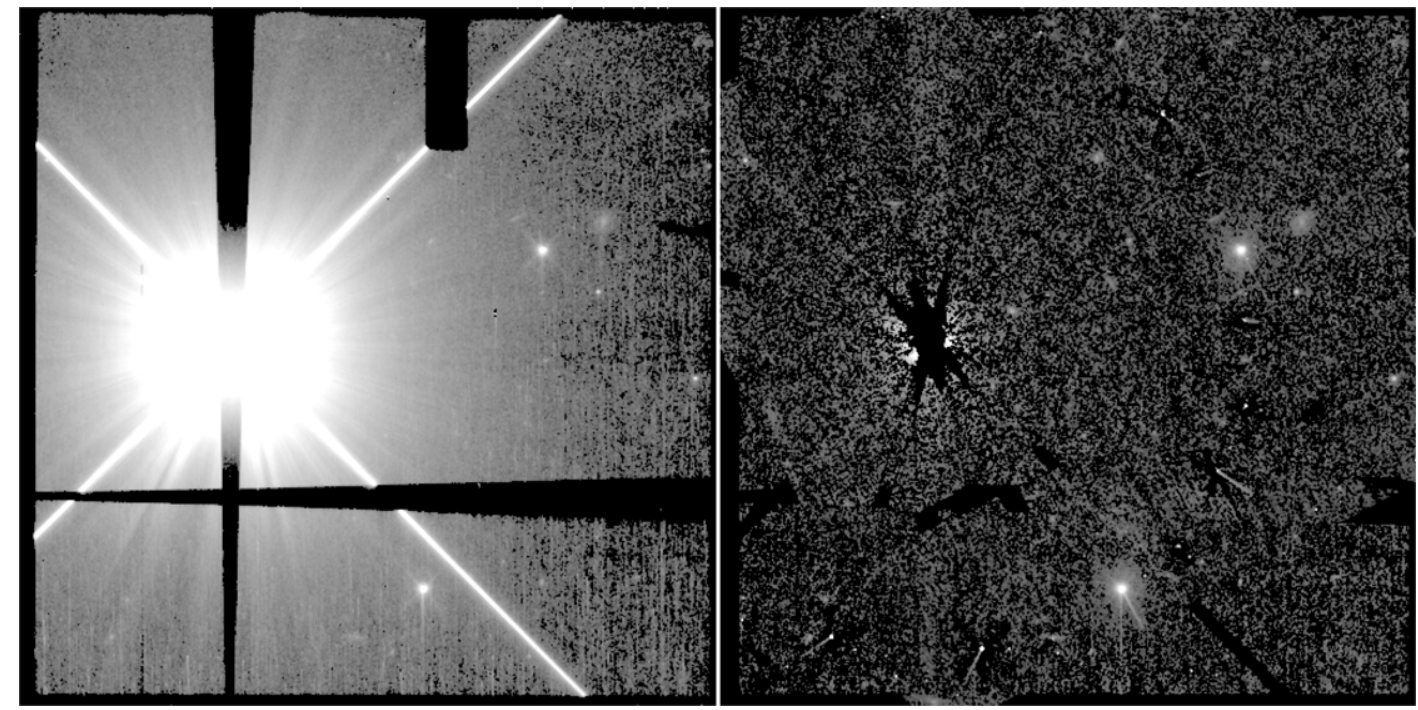

Figure 7. STIS coronagraphic images of HD 145229, both displayed with identical quarter-root intensity stretches. The images are 52" on a side. Left: PSF-unsubtracted image from the first orientation. The occulting mask shadows and diffraction spikes from the star are evident. Right: PSF-subtracted, combined images from the two orientations produced by the iterative roll subtraction algorithm.

Table 2

Debris Disk Non-detections in HST Program 12291

\begin{tabular}{lccclccc}
\hline \hline Target & $\begin{array}{c}\text { Spectral } \\
\text { Type }\end{array}$ & $\begin{array}{c}V \\
(\mathrm{mag})\end{array}$ & $\begin{array}{c}\text { Distance } \\
(\mathrm{pc})\end{array}$ & $\begin{array}{c}\text { Observation } \\
\text { Date }\end{array}$ & $\begin{array}{c}L_{\text {dust }} / L_{\star} \\
\times 10^{-4}\end{array}$ & $\begin{array}{c}\text { Spitzer } \\
\text { Reference }^{\mathrm{a}}\end{array}$ & Comments \\
\hline HD 377 & G2 & 7.6 & 39 & 2011 Jul 22 & 4 & H08 & NICMOS non-detection \\
HD 7590 & G0 & 6.6 & 23 & 2010 Oct 25 & 3 & P09 & Age 500 Myr \\
HD 38858 & G4 & 6.0 & 15 & 2011 Dec 24 & 1 & B09 & Disk resolved by Spitzer, has planet \\
HD 45184 & G2 & 6.4 & 22 & 2011 Oct 3 & 1 & K10 & Has planet \\
HD 73350 & G5 & 6.7 & 24 & 2011 Nov 24 & 1 & P09 & Age 500 Myr \\
HD 135599 & K0 & 6.9 & 16 & 2011 May 2 & 1 & P09 & Age 200 Myr \\
HD 145229 & G0 & 7.5 & 35 & 2011 Jun 3 & 1 & H08 & NICMOS non-detection \\
HD 187897 & G5 & 7.1 & 35 & 2010 Oct 10 & 1 & H08 & NICMOS non-detection \\
HD 201219 & G5 & 8.0 & 38 & 2010 Nov 2 & 1 & H08 & NICMOS non-detection \\
\hline
\end{tabular}

Note. ${ }^{a}$ B09: Bryden et al. (2009), H08: Hillenbrand et al. (2008), K10: Koerner et al. (2010), and P09: Plavchan et al. (2009).

and the outer extent of the disk. These will allow the use of modeling, as in Chiang et al. (2009), to more precisely constrain the location and mass of the perturbing planet.

Based on observations made with the NASA/ESA Hubble Space Telescope, obtained from the data archive at the Space Telescope Science Institute. STScI is operated by the Association of Universities for Research in Astronomy, Inc., under NASA contract NAS 5-26555. The Spitzer Space Telescope is operated by the Jet Propulsion Laboratory, California Institute of Technology, under NASA contract 1407. This work was funded by NASA via a Hubble Space Telescope General Observer Grant (program 12291) to the Jet Propulsion Laboratory, California Institute of Technology, and the Spitzer Project Science Office at JPL.

\section{APPENDIX}

Images of the other nine stars in our program (Table 2) revealed no signs of circumstellar nebulosity. The quality of the PSF subtraction for each was similar to that obtained for HD 202628. As an example, Figure 7 shows one of the candidates, HD 145229, before and after PSF subtraction.

To determine detection limits in these images, we followed the procedure used by Krist et al. (2010). They determined that a robust visual detection limit for an extended source (specifically, a $1^{\prime \prime} \times 1^{\prime \prime}$ uniform square) is 1.2 times the local rms noise level. In each of our roll-subtracted images, the rms of all pixels at each radius was computed, producing a radial noise profile. Visually identified bad (hot) pixels and background objects were omitted. The noise curves were multiplied by 1.2 and converted to surface brightness in $V$ magnitudes per square arcsecond (assuming neutral scattering). The conversion used the predicted count rate for each target provided by the STIS exposure time calculator based on the star's $V$ magnitude and spectral type. The results are shown in Figure 8. Rebinning by $2 \times 2$ pixels (to 0 ' 1 pixels) shifts these curves downward by $\sim 0.5 \mathrm{mag}$. The curves for the brightest stars are dominated by subtraction residuals at all radii, while for most targets the detection limit converges to $24 \mathrm{mag} \operatorname{arcsec}^{-2}$ at larger angles due to detector noise.

HD 202628 does not have any characteristics that distinguish it from the other stars, so there is no clear explanation why more disks in our sample were not detected. As noted before, a face-on disk would be self-subtracted out by the roll subtraction algorithm. However, the chances of any given disk being viewed face-on is low, and if one were, it would be difficult to detect regardless of the subtraction method due to the low integrated column depth (the most face-on disk seen in scattered light so far is HD 107146 (Ardila et al. 2004), with an inclination of 

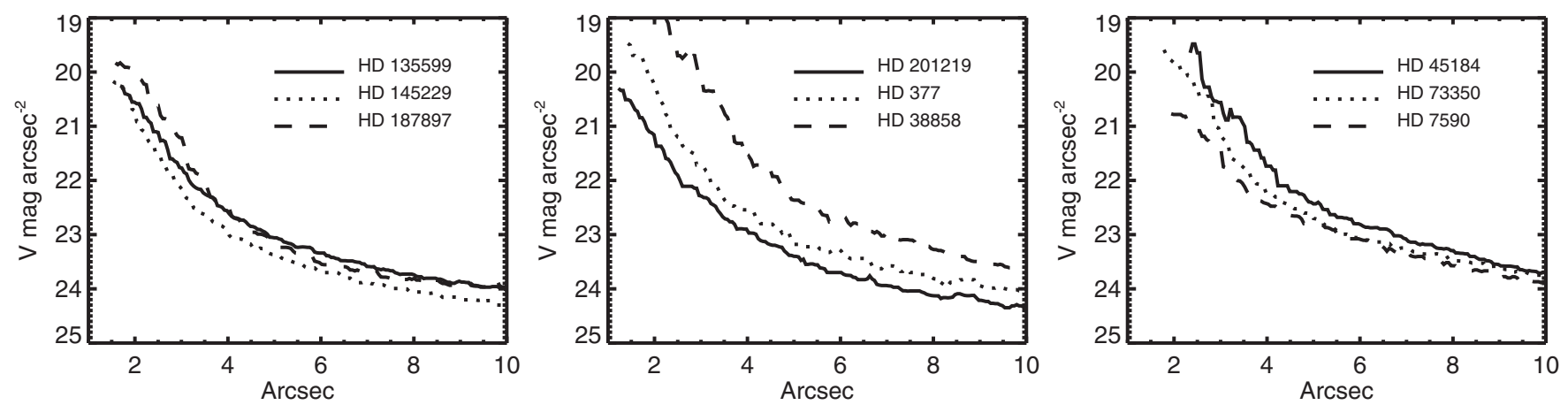

Figure 8. Lower surface brightness limits for the reliable visual detection of a $1^{\prime \prime} \times 1^{\prime \prime}$ square around targets for which no disks were seen.

$\sim 25^{\circ}$ from pole-on and an $L_{\text {dust }} / L_{\text {star }}=1.2 \times 10^{-3}$, which is about an order of magnitude brighter than our targets). Other causes are discussed in Krist et al. (2010).

Two of our targets are notable. HD 38858 has a Spitzerresolved $(70 \mu \mathrm{m})$ disk (G. Bryden et al. 2012, in preparation) with a radius of $9^{\prime \prime}$ (135 $\left.\mathrm{AU}\right)$, inclination of $48^{\circ}$, and the apparent major axis along P.A. $=56^{\circ}$ (our observations of this target were specified to place the disk major axis perpendicular to the occulting wedge). The star also has a radial velocity (RV) detected planet (HD 38858 b; Mayor et al. 2011) with a period of 407.1 days, semimajor axis $=1.04 \mathrm{AU}$, and $m \sin i=30.6$ $M_{\text {earth}}$. HD 45184 also has an RV planet (HD 45184 b; Mayor et al. 2011) with a period of 5.9 days, semimajor axis $=0.06 \mathrm{AU}$, and $m \sin i=12.7 M_{\text {earth }}$.

By themselves, these HST detection limits place weak constraints on the dust properties of the disks. However, when combined with resolved emission images of these disks obtained with Spitzer or Herschel, they provide very strong constraints on albedo and grain size distributions. We will be reporting these parameters for the non-detections from this and other HST programs.

\section{REFERENCES}

Ardila, D. R., Golimowski, D. A., Krist, J. E., et al. 2004, ApJ, 617, L147 Bryden, G., Beichman, C. A., Carpenter, J. M., et al. 2009, ApJ, 705, 1226
Chiang, E., Kite, E., Kalas, P., Graham, J. R., \& Clampin, M. 2009, ApJ, 693 734

Dodson-Robinson, S. E., Veras, D., Ford, E. B., \& Beichman, C. A. 2009, ApJ, 707,79

Ertel, S., Wolf, S., Metchev, S., et al. 2011, A\&A, 533, A132

Golimowski, D. A., Krist, J. E., Stapelfeldt, K. R., et al. 2011, AJ, 142, 30

Henry, T. J., Soderblom, D. R., Donahue, R. A., \& Baliunas, S. L. 1996, AJ, 111,439

Hillenbrand, L. A., Carpenter, J. M., Kim, J. S., et al. 2008, ApJ, 677, 630

Holmberg, J., Nordström, B., \& Andersen, J. 2009, A\&A, 501, 941

Hughes, A. M., Wilner, D. J., Andrews, S. M., et al. 2011, ApJ, 740, 38

Hünsch, M., Schmitt, J. H. M. M., Sterzik, M. F., \& Voges, W. 1999, A\&AS, 135,319

Kalas, P., Graham, J. R., Chiang, E., et al. 2008, Science, 322, 1345

Kalas, P., Graham, J. R., \& Clampin, M. 2005, Nature, 435, 1067

Koerner, D. W., Kim, S., Trilling, D. E., et al. 2010, ApJ, 710, L26

Krist, J. E. 2004, Proc. SPIE, 5487, 1284

Krist, J. E., Stapelfeldt, K. R., Bryden, G., et al. 2010, AJ, 140, 1051

Lebreton, J., Augereau, J.-C., Thi, W.-F., et al. 2012, A\&A, 539, A17

Marois, C., Macintosh, B., Barman, T., et al. 2008, Science, 322, 1348

Mayor, M., Marmier, M., Lovis, C., et al. 2011, arXiv:1109.2497

Plavchan, P., Werner, M. W., Chen, C. H., et al. 2009, ApJ, 698, 1068

Quillen, A. C. 2006, MNRAS, 372, L14

Schneider, G., Silverstone, M. D., Hines, D. C., et al. 2006, ApJ, 650, 414

Schneider, G., Weinberger, A. J., Becklin, E. E., Debes, J. H., \& Smith, B. A. 2009, AJ, 137, 53

Su, K. Y. L., Rieke, G. H., Stapelfeldt, K. R., et al. 2009, ApJ, 705, 314

Thalmann, C., Janson, M., Buenzli, E., et al. 2011, ApJ, 743, L6

Thébault, P., Marzari, F., \& Augereau, J.-C. 2010, A\&A, 524, A13

Valenti, J. A., \& Fischer, D. A. 2005, ApJS, 159, 141

Wyatt, M. C., Dermott, S. F., Telesco, C. M., et al. 1999, ApJ, 527, 918 